Result Over 180 group locations in different geographies completed self-assessment questionnaires (SAQs). Gaps were identified and improvement plans were executed at locations. This has resulted into overall improvement of WASH scores across the group from approx. $75 \%$ to $>90 \%$.

Discussion A focused approach of self-assessment questionnaire along with gap identification and action plans in a web based software has been found very useful to monitor and support development of WASH status across the organisation. This initiative has also resulted in increased awareness among workforce, peers and other stakeholders regarding WASH.

\section{WORK-RELATED ILL-HEALTH: REPUBLIC OF IRELAND, NORTHERN IRELAND, GREAT BRITAIN 2005-2016}

${ }^{1}$ Annemarie Money, ${ }^{1}$ Melanie Carder, ${ }^{2}$ Peter Noone, ${ }^{3}$ Johnny Bourke, ${ }^{4}$ James Hayes, ${ }^{1}$ Raymond Agius. 'University of Manchester, Manchester, UK; ${ }^{2}$ Health Service Executive, Dublin, Republic of Ireland; ${ }^{3}$ South Infirmary-Victoria Hospital, Cork, Republic of Ireland; ${ }^{4}$ Cavan Monaghan Hospital, Cavan, Republic of Ireland

\subsection{6/oemed-2018-ICOHabstracts.412}

Background Data on work-related ill-health (WRIH) in the Republic of Ireland (ROI) are inconsistent, with no mandatory requirement for employers to report occupational diseases/illness unless liable for compensation.

Aims To compare the incidence of WRIH in ROI, Northern Ireland (NI) and Great Britain (GB).

Methods Analysis of voluntary reported, medically verified data submitted to The Health and Occupation Research (THOR) network in ROI, 2005-2016. ROI-THOR comprises 4 schemes (74 physicians) enabling chest physicians (ROISWORD), dermatologists (ROI-EPIDERM), occupational physicians (ROI-OPRA) and general practitioners (THOR-GP-ROI) to report. Data were compared with the corresponding UK THOR schemes.

Results 2148 case reports (dermatologists: 453, chest physicians: 164, OPs: 1514, GPs: 17) were reported to ROITHOR. Contact dermatitis was the most frequently reported skin disease in all three areas (ROI, 96\%; NI, 48\%, GB, $76 \%)$. Asthma was the most frequently reported respiratory disease in the ROI (36\%), whilst for GB and NI it was benign pleural disease (42\% and 36\%, respectively). OPs and GPs in the ROI reported mental ill-health $(53 \%, 35 \%)$ and musculoskeletal disorders $(34 \%, 24 \%)$ most frequently; a similar pattern was observed in NI and GB. ROI skin and respiratory incidence rates (based on reports from dermatologists and chest physicians) were generally similar, or slightly lower compared to NI and GB.

Conclusions Overall, THOR-ROI continues to provide the best overall source of data relating to medically attributed occupational disease incidence in the ROI. Comparisons with UK data suggest specialists in the ROI see proportionately less long latency skin (e.g. neoplasia) and respiratory (e.g. mesothelioma; lung cancer) diagnoses compared to the UK. Other observed differences included a much larger proportion of OP ROI cases originating from the health and social care sector compared to GB and NI reports.

\section{RETURN TO WORKAND WORK PARTICIPATION AFTER CHANGES IN OCCUPATIONAL HEALTH SERVICE AND HEALTH INSURANCE ACT. NATIONWIDE FINNISH REGISTER STUDIES}

${ }^{1} \mathrm{E}$ Viikari-Juntura*${ }^{2}{ }^{2} \mathrm{U}$ VIrta, ${ }^{1} \mathrm{~T}$ Leinonen, ${ }^{1} \mathrm{~K}$ Husgafvel-Pursiainen, ${ }^{2} \mathrm{I}$ Autti-Rämö, ${ }^{1} \mathrm{~S}$ Solovieva. ${ }^{1}$ Finnish Institute of Occupational Health, Helsinki, Finland; ${ }^{2}$ The Social Insurance Institution of Finland, Helsinki, Finland

\subsection{6/oemed-2018-ICOHabstracts.413}

Introduction In many countries the social security legislation has been changed to support staying at work and return to work (RTW) from sickness absence (SA). However, the effectiveness of such changes is not well known. The aim was to assess the effectiveness of the introduction of part-time sick leave in 2007 and an amendment in its use in 2010 (enabling use at early stage of disability) on RTW and work participation. We also looked at RTW and work participation after the so-called 30-60-90 day rule was enacted in 2012, obligating, among others, early notification of prolonged SA (>30 days) as well as assessment of remaining work ability and possibilities to continue working (before 90 days).

Methods We used nationwide register information on ill-health benefits, as well as employment and unemployment periods. Receivers of partial sickness benefit were compared with propensity-score matched controls of full sickness benefit receivers. For the 30-60-90 day rule, we followed-up (2-10 months) those who had a continuous SA of 30 calendar or 60 compensated days before and after 2012.

Result Part-time sick leave at the early stage of disability enhanced return to work. Moreover, the proportion of time at work was at a significantly higher level in the part-time than full-time sick leave group. The prevalence of full disability retirement reduced and that of partial disability retirement increased among users of part-time compared with those with full-time sick leave. Work participation did not essentially differ after a SA of 30 calendar or 60 compensated days after the introduction of the 30-60-90 day rule.

Discussion The use of part-time sick leave enhances return to work and overall work participation, and should be considered, when a person is not able to work full time. The 3060-90 day rule seems not to have affected work participation during our follow-up times.

\section{SIGNATURE OF EPIGENETIC ALTERATIONS INDUCED BY CARBON NANOTUBE- IN VITRO, IN VIVO AND IN WORKERS}

${ }^{1}$ Lode Godderis*, ${ }^{2}$ Deniz Öner, ${ }^{2}$ Manosij Ghosh, ${ }^{3}$ Bram Bekaert, ${ }^{2}$ Ali M Tabish, ${ }^{2}$ Peter HM Hoet. ${ }^{1} \mathrm{KU}$ Leuven, Department of Public Health and Primary Care, Centre Environment and Health/idewe, Leuven, Belguim; ${ }^{2}$ Department of Forensic Medicine, Laboratory of Forensic Genetics and Molecular Archaeology, University Hospitals Leuven, Leuven, Belguim; ${ }^{3} \mathrm{KU}$ Leuven, Department of Public Health and Primary Care, Centre Environment and Health, Leuven, Belgium

\subsection{6/oemed-2018-ICOHabstracts.414}

Introduction Growing indication of toxicity and production of carbon nanotubes (CNTs), have resulted in concern about 